\title{
3D Sketching for Interactive Model Retrieval in Virtual Reality
}

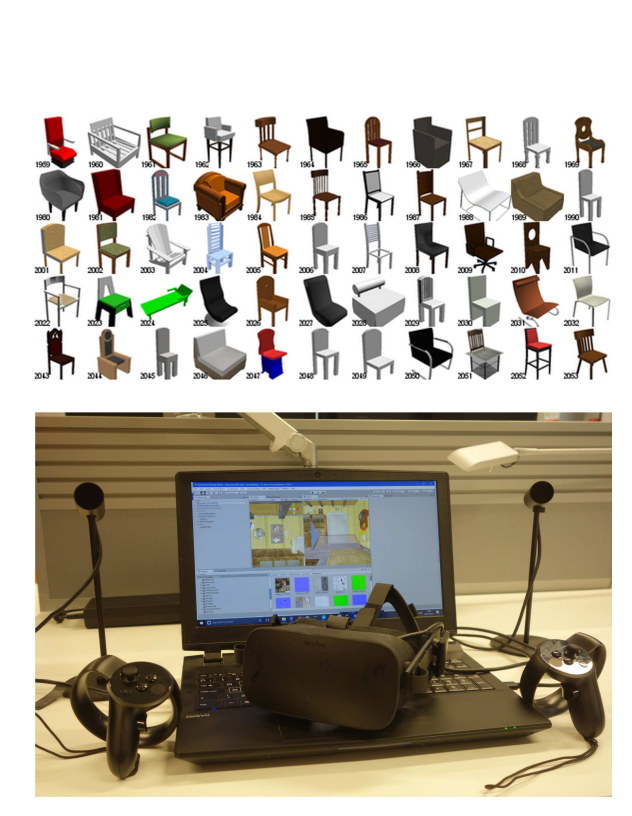

\author{
Anonymous Author(s)
}

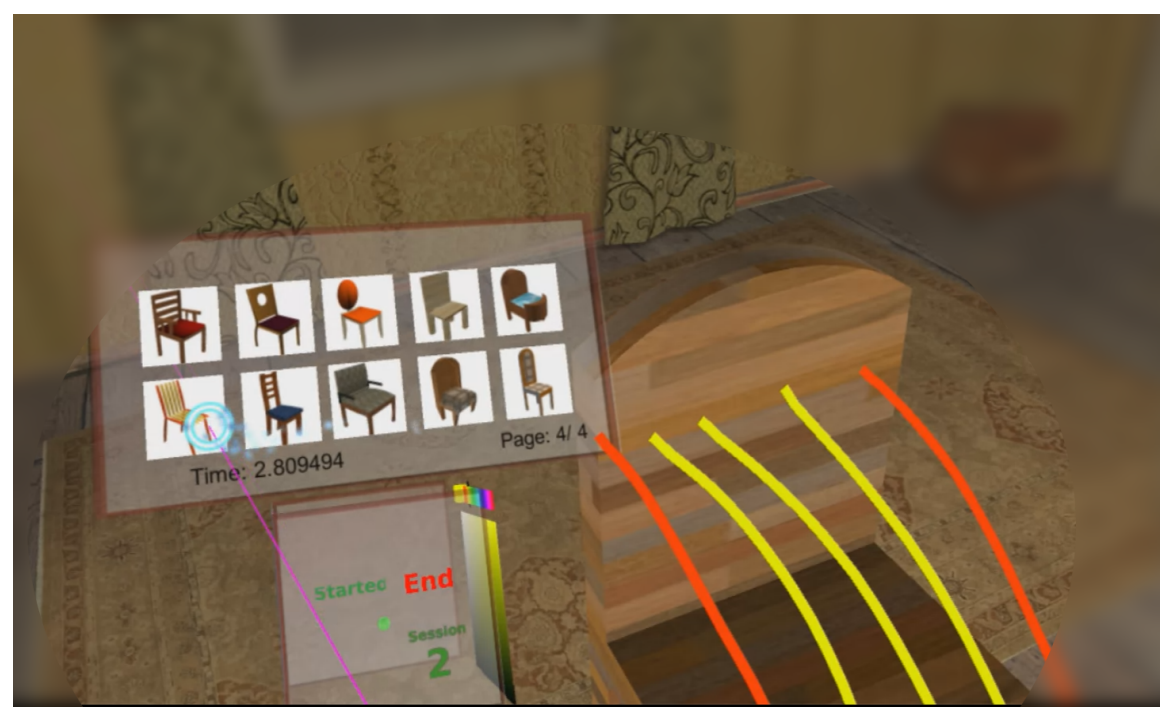

Figure 1: Our approach searches a dataset of chair 3D models (top left) using free-hand drawn sketches within a virtual reality. Our setup consists of Oculus RIFT, Oculus Touch and a laptop (bottom left). Sketches can be made either on top of a template chair or a prior search result (right).

\begin{abstract}
We describe a novel method for searching 3D model collections using free-form sketches within a virtual environment as queries. As opposed to traditional sketch retrieval, our queries are drawn directly onto an example model. Using immersive virtual reality the user can express their query through a sketch that demonstrates the desired structure, color and texture. Unlike previous sketch-based retrieval methods, users remain immersed within the environment without relying on textual queries or $2 \mathrm{D}$ projections which can disconnect the user from the environment. We perform a test using queries over several descriptors, evaluating the precision in order to select the most accurate one. We show how a convolutional neural network $(\mathrm{CNN})$ can create multi-view representations of colored 3D sketches. Using such a descriptor representation, our system is able to rapidly retrieve models and in this way, we provide the user with an interactive method of navigating large object datasets. Through a user study we demonstrate that by using our VR 3D model retrieval system, users can perform search more quickly and intuitively than with a naive linear browsing method. Using our system users can rapidly populate a virtual environment with
\end{abstract}

Permission to make digital or hard copies of part or all of this work for personal or classroom use is granted without fee provided that copies are not made or distributed for profit or commercial advantage and that copies bear this notice and the full citation on the first page. Copyrights for third-party components of this work must be honored. For all other uses, contact the owner/author(s).

Expressive 2018, August 2018, Victoria, British Columbia, Canada

(c) 2018 Copyright held by the owner/author(s).

ACM ISBN 123-4567-24-567/08/06.

https://doi.org/10.475/123_4 specific models from a very large database, and thus the technique has the potential to be broadly applicable in immersive editing systems.

\section{CCS CONCEPTS}

- Human-centered computing $\rightarrow$ Virtual reality; HCI design and evaluation methods; • Computing methodologies $\rightarrow$ Object recognition; Ranking;

\section{KEYWORDS}

Sketch, Virtual Reality, CNN, HCI

\section{ACM Reference Format:}

Anonymous Author(s). 2018. 3D Sketching for Interactive Model Retrieval in Virtual Reality. In Proceedings of Expressive (Expressive 2018). ACM, New York, NY, USA, 11 pages. https://doi.org/10.475/123_4

\section{INTRODUCTION}

In recent years, with the rapid growth of interest in 3D modeling, repositories of 3D objects have ballooned in size. While many models may be labeled with a few keywords and/or fields that describe their appearance and structure, these are insufficient to convey the complexity of certain designs. Furthermore, in many existing databases, these keywords and fields are incomplete. Thus queryby-example methods have become a very active area of research. In query-by-example systems (see Section 2), the user typically sketches elements of the object or scene they wish to retrieve. A search system then retrieves matching elements from a database. 
In our system, the user is immersed in a virtual reality display. We provide a base example of the class of object to act as a reference for the user. The user can then make free-form colored sketches on and around this base model. A neural net system can analyze this sketch and retrieve a set of matching models from a database. The user can then iterate by making further correctional sketches (e.g. adding new pieces to the model) until they find an object that closely matches their intended model. This leverages the strengths of traditional approaches while embracing new interaction modalities uniquely available within a $3 \mathrm{D}$ virtual environment.

The main challenge in sketch-based retrieval is that annotations in the form of sketches are an approximation of the real object and may suffer from being a subjective representation and oversimplifications. These abstract representations present challenges to description methods and therefore require unique consideration. For image retrieval, methods focus on enhancing lines through gradients, GF-HOG [25] and Tensor Structure [19], with more recent approaches based on convolutional neural networks (CNNs) [9, 55]. In contrast for $3 \mathrm{D}$, the use of sketching for retrieval has been limited to $2 \mathrm{D}$ projections for matching [20]. To match $3 \mathrm{D}$ models, it is typical to normalize the models to have the same orientation, so that a standard set of images at set orientation can be rendered to compare the sketch to (see Section 2.2.2). We adopt this view-based method as it allows an interactive experience where users can get responses with little time delay.

So far, sketching within a virtual environment as a retrieval method has received little attention. There are various tools to allow the user to sketch (e.g. Tiltbrush, or Quill), but these focus on the sketch itself as the end result. Other systems allow free-form manipulation of objects by simple affine manipulation through drag points [42]. In contrast, we instead are interested in how a user can utilize sketch as a method of retrieval. We therefore performed a user study to compare sketch-based retrieval to a naive linear browsing to demonstrate that sketching is an effective and usable method of exploring model databases.

The contributions of our work are fourth-fold. First, we present a novel approach to searching model collections based on annotations on an example model. This example model represents the current best match within the dataset and sketching on this model is used to retrieve a better match. A novel aspect of our method is that we allow users to make sketches directly on top of existing models. The users can express color, textures and the shape of the desired object. Second, We evaluate different descriptors through a preliminary study in order to select the most accurate one, discovering that $\mathrm{CNN}$ achieves the highest precision. Third, we perform a user study to demonstrate the advantages of a sketch-based retrieval system in contrast to naive search. We show that users understand the purpose and practical use of a sketch-based retrieval system and that they are easily able to retrieve target objects from a large database. Finally, our system is the first of its type to work online in an immersive virtual environment. This model retrieval technique can be broadly applied in editing scenarios, and it enables the editor to remain immersed within the virtual environment during their editing session.

In the remainder of the paper, Section 2 reviews virtual environment modeling, sketching and representation methods. Section 3 explains the intricacies of our virtual environment model retrieval system and the novel use of interactive machine-learning-based searches to enable an iterative sketch and query refinement process. Section 4 presents a user study to demonstrate it's effectiveness in terms of both accuracy and user experience rating. We then discuss the comparison between descriptors and the search technique in Section 5 and describe future work and limitations in Section 6. We then conclude in Section 7.

\section{RELATED WORKS}

Sketching represents a natural way for people to convey information. Eitz et al. [21] give an overview of how people sketch objects and how sketches are recognized by humans and computers. The fundamental supposition is that sketches approximate the real world object. On the other hand, since the average user is not an artist, the subjective representation of an object can be iconic and include possible simplification of the objects. We explore the implications of this for both retrieval and interaction with respect to both 2D (Image) and 3D domains.

\subsection{Sketch-based Image Retrieval}

Identifying and associating a sketch with a specific object in an image represents a hard challenge. However, it is an attractive strategy because the use of sketch interaction is an opportunity to broaden the user base to those who are unfamiliar with complex interactive editing systems. Various methods for retrieving images from sketches have been developed. These systems are referred to as sketch-based image retrieval (SBIR) systems [6]. SBIR techniques can be classified into two classes: blob-based techniques that focus the attention on features such as shape, color or texture, and contour-based techniques that describe the image using curves and lines. Techniques belonging to the blob-based SBIR class try to describe image through descriptors such as QBIC [3] which use separately color, texture and shape or [46] which uses topology models. Contour-based techniques include elastic matching [5] and grid and interest points such as edge points [12].

In recent years researchers have applied machine learning algorithms to SBIR. SketchANet [54] is a simple neural network based on Alexnet that performs sketch recognition. Qi et al. [37] introduce a siamese CNN which aims to measure the compatibility between image edge-map and sketch used as CNN inputs. Collomosse et al. [10] did a review of different triplet $\mathrm{CNN}$ architectures for evaluating the similarity between pictures and sketches, focusing on the capacity to generalize between object classes. Triplet architectures (Wang et al. [50], Sangkloy et al. [41]) have attracted increasing attention for the relationship of the three branches when processing the loss function: firstly the anchor branch (modeling the reference object), secondly a branch which models positive examples and thirdly a branch that deals with negative examples.

A strategy to improve the performance of image retrieval systems is to put the user 'in the loop' and take advantage of iterative refinement. This technique is called relevance feedback in information retrieval and was introduced in Content-Based Retrieval by Sciascio et al. [44]. Several applications based on interactive sketch systems have been created. For example, Shadow Draw of Haldankar et al. [31], iCanDraw [16], Sketch-to-Collage [39] and CALI system from Fonseca et al. [27]. 


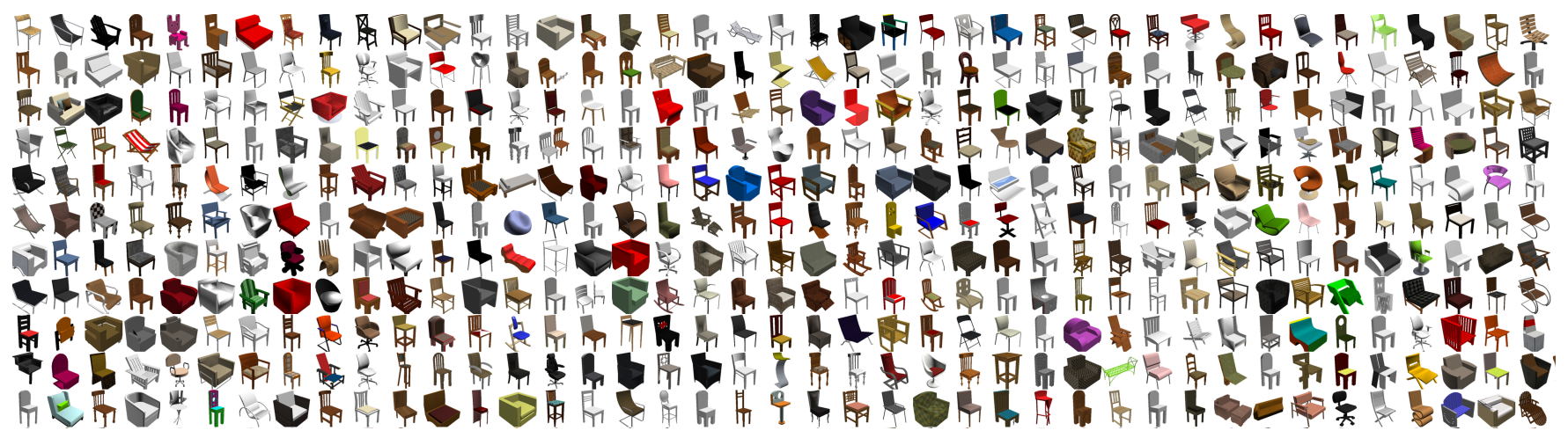

Figure 2: A collage of the chairs class models from ShapeNet dataset[52]. The collage shows a subset of the total set of 3370 chairs in order to illustrate the diversity of this class of object.

\subsection{D Sketch-based Retrieval and Interaction}

Finding features that represent $3 \mathrm{D}$ objects is a unique challenge in the retrieval domain. Since one of the most important cues in object recognition is $3 \mathrm{D}$ geometric shape, sketching in 3D could represent a problem due to the abstract nature of the sketch. In addition, before sketch interpretation, a simplification process of the stroke can be taken for avoiding noisy samples [23] since both the tracking device and user generate noise during sketch acquisition.

In recent years, researchers have proposed several descriptors for $3 \mathrm{~d}$ view-based that can be classified into two categories: modelbased and view-based.

2.2.1 Model-based descriptors. Measuring similarities between $3 \mathrm{D}$ models is a hard problem. Object models can differ in shape, color and orientation in 3D space, making the definition of a similarity measure challenging. Different categories of descriptors were created to overcome this challenge: geometric moment, surface distribution and volumetric descriptors. Geometric moment [8] is a class of topology invariant similarity methods based on vector coefficient extracted by a shape decomposition under specific basis. Surface distribution [36] tries to measure the global properties through a shape distribution achieved by sampling a shape function and in this way reduces a shape comparison to a simpler distribution comparison. Volumetric descriptors [40] combine shape distributions with barycentroid potential for achieving a more robust pose and topology invariant similarity. Despite the extensive research on descriptors that allows extracting shape characteristics, only with the advent of deep learning architectures such as Restricted Boltzmann Machines (RBM), Deep Belief Networks (DBN) and Deep Boltzmann Machines(DBM), and in particular CNN [30] have achieved a relevant improvement of outcomes in object recognition. Wu et al. [52] recently proposed a method to represent a 3D object through the distribution of binary variables in a volumetric grid, and use of Convolutional Deep Belief Networks to extract features and recognize them.

2.2.2 View-based descriptors. View-based descriptors use 2D projections of the objects from different points of view. Since a large amount of information that can be collected in this way, these methods outperform model-based descriptor approaches. Ansary et al. [2] introduce a model-index technique for 3D objects that make uses of $2 \mathrm{D}$ views. It uses a probabilistic Bayesian method for 3D model retrieval. Alternatively, Su et al. [47] present a framework using view-based descriptors, creating 12 views for each object that feed a first CNN for feature extraction, and after a pooling stage, the results are passed to another $\mathrm{CNN}$ for achieving a compact shape descriptor. Similarly, Leng et al. [32] proposed a 3DCNN that manages multiple views and considers possible interactions between them. In a pre-process stage a sorting algorithm, which takes in consideration the angles and positions, prepares three different sets of viewpoints and the network is fed with them at the same time. This is a different approach from the classic one which uses only one view at a time and it confers stability during the training stage.

Our method uses view-based descriptors, rather than modelbased descriptors because they have demonstrated more practical utility on similar problems.

2.2.3 3D sketching. Considering alternative uses of sketch interaction within a 3D context, Wang et al. [49] present a minimalist approach in terms of view-based descriptors. They generate only two views for the entire dataset and train a Siamese CNN with the views and the sketches. Nishida et al. [35] proposed a novel method to design buildings from sketches of different parts of them. The user sketches few strokes of the current object and through a pre-trained $\mathrm{CNN}$ for that specific object type, the system is able to procedurally retrieve the correct grammar snippet and select the most similar one. The final step of the process is to combine all the snippets in a unique grammar of the building just created.

3D sketch-based model retrieval has gained significant attention in recent years. Funkhauser et al. [24] proposed a combination of sketch and text query to identify 3D objects. They showed that the combination of the two methods results in a better accuracy of the results. Shin and Igarashi [45] with Magic Canvas provided the user with a system for 3D scene construction using sketches, based on sketch-object similarity. In addition, the system determines the position and orientation of the object according to the sketch. $\mathrm{Xu}$ et al. [53] with Sketch2Scene proposed a novel framework for scene modeling through sketch drawing that suggests also the placement of the objects via functional and spatial relationship 
between objects. Critically these methods have generally used 2D sketches. Our system allows the user to sketch in 3D.

2.2.4 Immersive Sketching. Immersive sketch-based modeling has gained a lot of attention over the years. A very early example is Clark's 3D modeling system for a head-mounted display [15]. The system of Butterworth et al. [11] supported several geometric modeling features, including freehand operations. More recently many immersive 3D modeling systems have exploited freehand sketching such as BLUI [7], CavePainting [28], Drawing on Air [29], FreeDrawer [51], Holosketch [18] and Surface Drawing [43]. Very recently applications for consumer virtual reality systems such as Tiltbrush from Google and Quill from Facebook have raised awareness of sketching for content development. The most similar work to ours is the system Air Sketching for Object Retrieval [4]. This combines 3D sketch and a search engine based on the spherical harmonic descriptor. Our system uses a different type of lightweight sketching over basic models and a view-based descriptor. Another similar system that is that of Li et al. [33], a content retrieval system that can benefit from sketch-based interaction using a Microsoft Kinect. Possibly because the sketches are relatively crude, they focus on distinguishing between classes of object. We focus on precise sketching to distinguish between similar objects in a large class of objects. Also, we enable sketching over existing models, rather than sketching from scratch.

\section{3D SKETCH-BASED RETRIEVAL DESIGN}

Sketch-based retrieval has had great success within 2D image retrieval, yet still is cumbersome when extended to 3D. We propose that by utilizing recent advances in virtual reality and by providing a guided experience, a user will more easily be able to retrieve relevant items from a collection of objects. We explore the proposed methodology on ShapeNet [52]. ShapeNet is an extensive 3D model collection that includes a large set of model classes. We demonstrate our method to the subset of this collection that contains chairs, although our method is applicable to many classes of object. The chair subset is large and exhibits a large amount of variation that is particularly suitable for our method (see fig. 2). We first outline our proposed Sketch-based Retrieval pipeline (subsec. 3.1) then go on to define a study to demonstrate the benefits of using such a method compared to naive linear searching (sec. 4).

\subsection{D Sketch-based Retrieval}

Searching for a model in a large collection using 2D sketches can be tedious and require an extended period of time. It also requires a particular set of skills, such as understanding perspective and occlusion. By using virtual reality this experience can be improved because ambiguity between views is greatly reduced and the user no longer has to hallucinate the projections from $2 \mathrm{D}$ to $3 \mathrm{D}$.

3.1.1 3D Sketch Descriptor. Sketching within a 3D environment has been explored through stroke analysis[14, 22, 38], but little work has been performed to describe the set of strokes in a compact representation, i.e. descriptor, such as in SBIR[21] or SBVR[26]. Therefore we explore state-of-the-art model descriptions approaches. We apply four traditional Bag of Words approaches: SIFT[34],Histogram

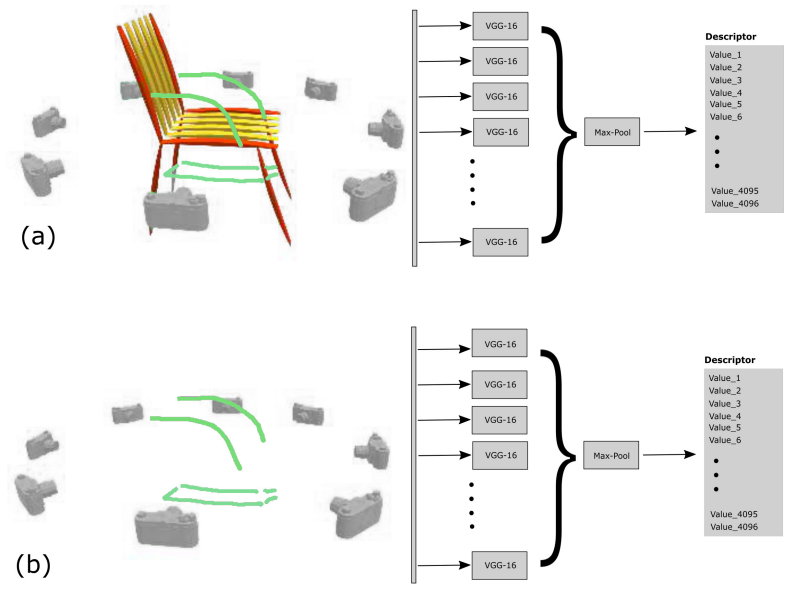

Figure 3: (a) CNN can be triggered with snapshots with both sketch and chair model. (b): CNN can be triggered with snapshots with only sketch present.

of Gradients(HoG)[17],Gradient Field Histogram of Gradients (GFHoG)[25] and ColorSIFT[1]. It is worth noting that only ColorSIFT descriptor incorporates a description of color. In addition, we apply a multi-view CNN architecture to describe the content of the model.

Each proposed method generates a unique descriptor of the chair. To generate a single vector description of a model the chair is projected into 12 distinct views as shown in fig. 3 . Each view is then described by an independent model. This exhibits an early fusion approach which we describe for both deep and shallow (BoW) descriptor generation methods.

In the multi-view CNN architecture[48] the standard VGG-M network of [13] is applied. This model consists of five convolutional layers and three fully connected layers (depicted in fig. 4). As in [48] the model is trained on ImageNet then fine-tuned on the 2D images of the 3D Shapes dataset. For each view of the model the convolutional layers of the VGG-M are applied where the resulting descriptors are aggregated by element-wise max pooling. The result of the max-pooling is then fed through a second part of the VGG-M network (i. e. $f c$ layers) where the second fully connected layer $\left(f c_{7}\right)$ is used as the descriptor for the view $(V)$ resulting in $V \in \mathbb{R}^{4096}$. The VGG-M network is trained once and shared amongst views.

For SIFT, ColorSIFT, HOG and GFHOG, we used the bag of words (BoW) mechanism to generate a descriptor from all the views. The BoW implementation is defined with $K=1024$ clusters that represent the visual words and where the frequency histogram across views is accumulated to generate a singular descriptor, $V \in \mathbb{R}^{1024}$ for these methods.

We perform a preliminary evaluation of the descriptors for retrieval of models (See Section 5.1) and identify the approach of Su et al. [48] to significantly outperform the alternative methods, henceforth we discuss the approach in regards to this descriptor. An index is generated from the dataset by repeating the aforementioned process over the dataset generating a matrix $M=D^{n \times 4096}$ where $n$ is the number of items in the collection. 


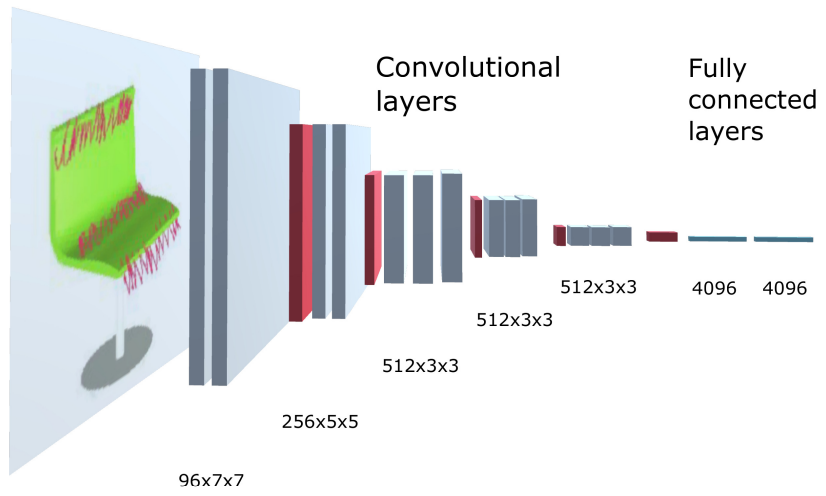

Figure 4: Each view is processed by the shown VGG-M architecture model [13]. As demonstrated in figure 3 the network is split after convolutional layers the final Multi-View descriptor is the output of the network a vector of 4096 scalars.

3.1.2 Online Queries. At query time, the multiple views are generated from the user's sketches and, optionally the current $3 \mathrm{D}$ model that is the best match (see below), and a forward pass through the network returns the descriptor. For simplicity and ease of comparison of results, we leave $M$ to be linearly searched at query time. Improved efficiency could be achieved by using KDTrees or other popular index structures. Therefore, we define the distance $d$ as squared Euclidean:

$$
d_{i}=\left|M_{i}-Q\right|^{2}
$$

where $\mathrm{Q}$ is the query descriptor. After comparing the descriptor with the descriptor collection the system replies with the $K$-nearest models that fit the input sent. In our experiments we use $K=40$. The retrieved models are ordered by their respective $r_{i}$ distance.

We provide the user two ways to perform the query: sketchonly query or both sketch and model query. This is achieved by enabling or disabling the visualization of the model (see figure 3). After the system proposes results, if the user's target model is not present the user can edit the sketch or conversely can replace the current model with a new one that better matches represents the desired target. Such a possibility helps the user to minimize the time sketching: they can focus on sketching on the missing or different parts relative to the current best match model. This facilitates a step by step refinement to navigate through the visual feature space of the collection, commonly achieving the target model only after a few iterations. In the current implementation (see Section 4.3) the response time after each user search request is 2 seconds. This is sufficiently quick to allow a tight interactive loop between sketching and querying. Users are free to either make a complex sketch that will likely match on the first attempt, or add features to the model in several iterations, thus facilitating a 'walk' through the model collection towards the desired target.

\section{USER STUDY: COMPARISON OF SKETCHED QUERIES OVER LINEAR SEARCH IN VR}

\subsection{Task Overview}

We designed an experiment to compare two methods: the proposed sketch-based method, and a naive scrolling panel method. For each session of the test, we first showed the participant the twelve views of a target chair as generated for the descriptor. We then asked the participant to retrieve the chair from the database, using one of the two methods. For both methods the participant started in a scene of a furnished room where a chair is positioned on the floor to the user's left-hand side. We perform this initialization step to minimize the required hand travel distance avoiding any mobility bias. We tracked the success rate, the time to complete the task and a subjective evaluation of the user experience through a questionnaire.

The scroll method consists of finding the target chair from the entire collection of 3370 chairs using a panel that shows 10 chairs at once and which can be scrolled forward and backward very quickly. After the user starts the session, the chairs are randomly shuffled to prevent recall of the order from memory. The user then simply searches for the target chair (see fig. 5). When the user is confident that they have found the chair, they select it from the panel in order to replace the current chair in the room. When the participant clicks the end label, the time required to complete the task is taken and the session is finished. For the sketch method, the user makes colored sketches on top of the initial model (see fig. 6) and then uses the hand-held device to trigger the search method. The system proposes 40 chairs as outcome, shown 10 at a time in a scroll panel which is navigable in the same fashion as the scroll method. The participants would iteratively sketch, triggering the retrieval system or selecting models from the 40 suggestions then continue to refine the sketch. The search refinement process continues until the target chair is located and the user can terminate the session.

\subsection{Procedure}

All participants are asked to complete an introduction form with basic information related to their previous user experience in 3D software and VR applications. Each user performs two sessions of tests. Where each session is comprised of two sub-sessions. In each sub-session, the user performs three search tasks for different

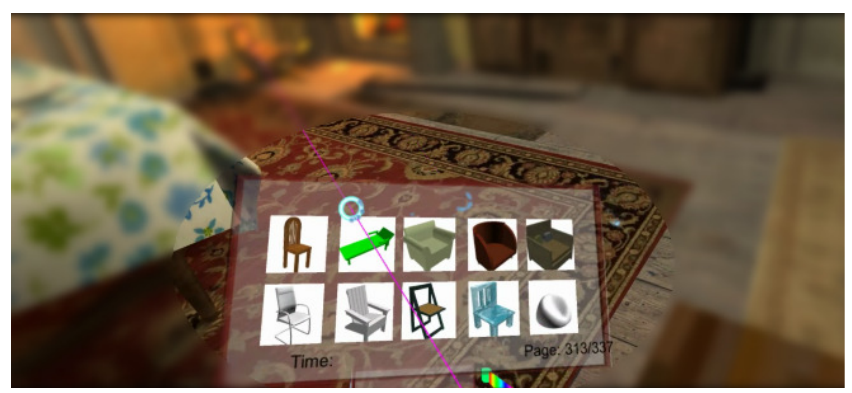

Figure 5: The scroll method provides a simple scrolling panel for navigating the database of all the chairs. 


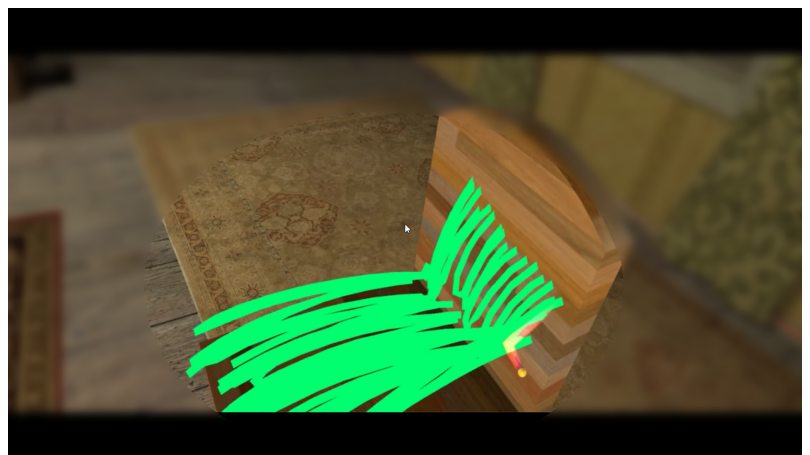

Figure 6: An example of a user's sketch within the sketch interface. The query is comprised of colored 3D strokes drawn on top of a chair model.

chairs models with one method, and then the same three searches with the other method.

Participants were instructed before each of the four sub-sessions with an application demo in which it will be shown the modality they had to use. In addition, they could select to practice for a short time to familiarize with the interaction. Each of the search tasks was started by asking the user to look at a particular target chair with the instruction find it using the selected method. For the sketchbased method, we instructed the user to use the style they prefer, that could be based predominantly on making a single sketch or on system interrogation with multiple iterations of model replacement. Each user was allowed to perform the task seated or standing. An upper time limit was defined as 4 minutes and in the event the user was unable to locate the target chair within the time limit or the wrong chair was selected, the search was considered a failure and the time cropped to 4 minutes. The two sessions differed in starting method used and from the different set of target chairs; thus the order of the methods is counter-balanced, and each subject uses both methods twice. We split the users into two groups: the first group started with the naive scroll method in the first session, while the second started with the sketch method. In total each participant performed 12 searches. In this way, we were able to analyze the task completion time considering the contribution related to the different techniques, to the chair types and to the learning curve effect of VR interaction. We choose six different chairs with specific structure and colors. In particular, both striped and curvy shapes are present in the sets with a variety of different colors as shown in fig. 8 . After completing all four sub-sessions (12 search tasks), participants filled in a final form with their rating on user experience and level of confidence for both scroll method and sketch method. The scale of the rating was expressed in the form of a scalar from 1 to 5 .

\subsection{Implementation}

The participants used an Oculus Consumer Version 1 (CV1) headmounted display (HMD) as well as Oculus Touch controllers. The experiment was performed on a PC laptop with a Processor Intel(R) Core(TM) i7-6700 CPU, NVIDIA GeForce GTX 980M graphics card and $64 \mathrm{~GB}$ of RAM.
The virtual reality software was created within Unity. The scene consisted of a furnished room, with the addition of a chair when the system was initialized. During the scroll-based method, the user can select models from a floating panel in which can scroll pages of models and display 10 models at time. The panel is attached to the left hand and the selection is performed using right-hand controller. 10 models were chosen so as to provide a panel that was small enough not to occlude large parts of the environments, but large enough that features in the chair were easily legible inside the HMD.

The sketching mechanism is implemented through the generation of colored lines. Lines are implemented as narrow strips that expose their wider section to the current camera. Therefore, each virtual camera, used for multi-view generation, renders the larger section of the strip independent from the sketch path. The user can the color using a palette connected to the left-hand GUI. The user can draw 3D lines in the virtual environment on top of the current model and can submit to the system using the controller's triggers. We provided also a simple UNDO function that acted on the sketch stack. We didn't provide additional tools in order to stimulate users to play essentially with pure sketch interaction. The back-end is a separate service thread in which a CNN Model is preloaded and ready to respond to user queries. We integrated the VGG-M Matlab implementation of Su et al. [48]. This is triggered to produce a unique visual descriptor given the snapshots generated by VR application as described in sec.3.1.

To maintain a reasonable computation time, the first convolutional layers (see figure 4) use stride 2, while the latter layers are used as normal. On average the $\mathrm{CNN}$ process takes approximately 0.5 seconds to produce a descriptor after receiving input.

\section{RESULTS}

In this section we describe the results achieved by preliminary study that compares different descriptors followed by the outcomes of the user test.

\subsection{Comparison between 3D Sketch Descriptors}

We perform a preliminary study using a set of six queries over the different descriptors and evaluate their retrieval precision with regards to a set of criteria for the returned model. Following the approach of Collomosse et al. [16, 26] we evaluate the precision in terms of of this different facets of the retrieval, therefore for each correctly returned facet of the model the score is incremented. These correspond to: 1) Structure - majority of the parts arms back, seat, legs; 2) Style - curvy, straight, with many lines; 3) Color dominant color matches query.

This study aims to identify the descriptor that achieves the best precision for the search task. The most accurate method is then used in the user test. In addition we prepared two sets of queries, the first are pure sketch queries, while the second are a combination of the sketch and the model. We considered the top 10 retrieved chairs proposed by each method, ranked from position 1 to position 10. Each rule can assign only one point if matched and focuses on a specific feature of the model. We formalized the rules as follows: 


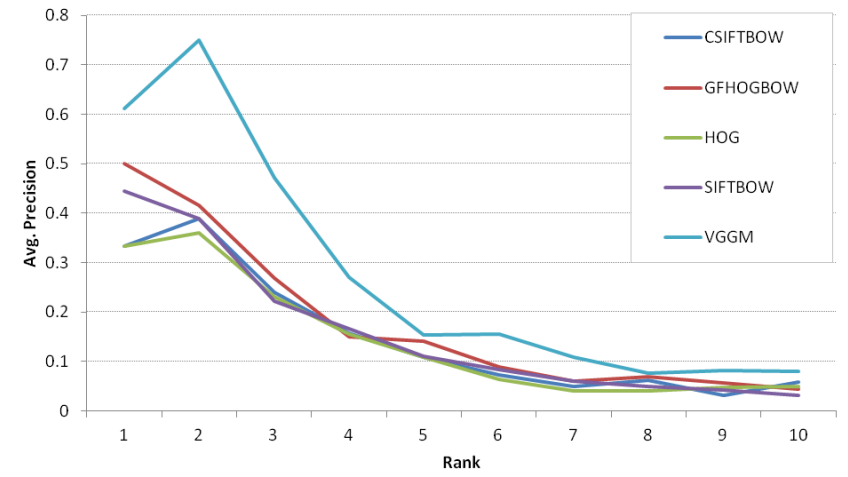

(a)

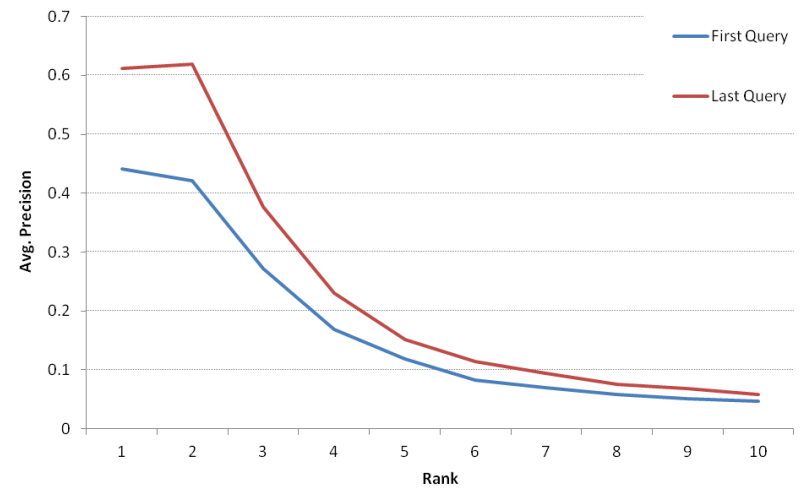

(b)

Figure 7: (a) Average precision calculated across ranked results from preliminary study. (b) Comparison of the first and last query average precision from user study.

(1) we consider 4 components of the chair: back, seat, arms and legs. Where if $\geqslant 75 \%$ are similar to the target, the result is considered correct;

(2) if the proposed chair shows a dominant style (curvy, stripes, convex, etc...) similar to the target chair;

(3) if the proposed chair shows a dominant color similar to the target chair.

With each result receiving points for the facets a final score in the range of [0,3] is calculated, which is then normalized across facets and queries for a result in $[0,1]$. The precision is calculated from the scores for each result, using the equations:

$$
P_{r}=\frac{\sum_{i=1}^{r} S_{i}}{r},
$$

where $P_{r}$ is the average precision for the rank $r, S_{i}$ is the score for rank $i$ assigned by our metric. We compare SIFT, ColorSIFT, HOG, GF-HOG and VGG-M, calculating the average precision for each chair of the top 10 retrieved models. VGG-M method outperforms all the other methods using sketch and model queries( as shown in fig. 7a) and also using only sketches (as shown in supplemental material).

We calculate Mean Average Precision (MAP) for each descriptor. For the sketch and model queries VGG-M's MAP achieves 0.28, followed by GF-HOG with 0.18 . This pattern is similarly reflected within the Sketch only queries, with VGG-M's MAP highest at 0.22 , followed by SIFT with 0.13 . Therefore, we perform the user test using descriptors generated by VGG-M.

\subsection{User Study}

Our user study consists of 30 participants recruited from the ANON department and general public. We split the participant into two equal size groups (15 users per group). The first group of participants started with scroll method, while the second group started with the sketch method. Twenty of the participants were male (10 female) while the average age of the participants is 26 years old. Each of the participants in the study performed 6 scroll and 6 sketch tasks, giving a total of 360 search tasks across all participants.
The tasks splits are demonstrated in figure 8 with regard to group and session (see supplementary material for user final queries). i. e. twelve trials per user, with fifteen participants doing the first task with scroll, fifteen doing the first task with sketch.

The number of successful task completions for the scroll method was 119 out of 180 (66\%) and for the sketch method 171 out of 180 (95\%). In fig. 13 we show the total number of completions for each method for each task, in the order that participants completed the tasks in their respective groups. This graph shows the impact of individual tasks being found easier or harder by the participants. As there does not appear to be a trend over the sequence of tasks for the sketch method, it demonstrates minimal learning required and the intuitive nature of the method.

The task completion performance for the sketch method can be affected by the complexity of the target model, where difficult models are challenging to depict. The participant may have improved their depiction ability or efficiency with the system, but this can not be conclusively drawn from these results. While the significant factor for the linear search is the position within the dataset. It also can be seen a much larger variation in completions per task for scroll than sketch. For task three only 3 participants completed the search with the scroll method. This in comparison with sketch, the minimum number of completions was 12 .

We show the time to complete all tasks in figure 10 for each of the methods. We can see that the distributions are very different, with a cross-over point at around 60 seconds. This can be explained by the fact that the completion time for the scroll method is largely determined by the page number that the result appears on; while for the sketching method there is an additional interaction overhead for completing the query sketch and the search time.

By comparing the average time to complete all six models for the sketch or scroll methods in a paired-comparison per user - i. e. each pair comprised the average time to complete all six sketch tasks and the average time to complete all six scroll tasks. Additionally where any failures to complete were clamped to 240 s (4 minutes). The median time to complete the sketch tasks was $99.8 \mathrm{~s}$, and the 


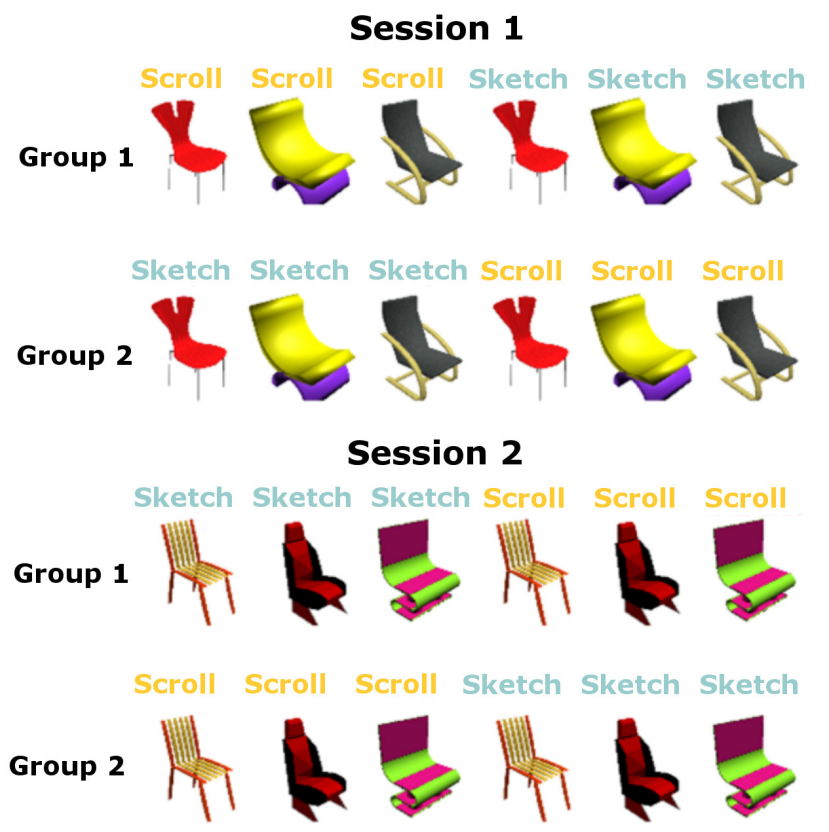

Figure 8: Two groups of 15 users are created. The first group performed the scroll method as the first method for the first set of chairs, then with the sketch method for the first set of chairs, then swapped the methods over for the second set of chairs. The second group did the opposite order of methods on the same order of sets of chairs.

median time to complete the scroll tasks was 156.5s. Because of the distribution of times, and the clamping on failure, we used the exact sign test to compare the differences. This showed that the difference in medians was significant, with p less than 0.0005 We asked participants to report a feedback on user experience. In fig. 11 we show an average rating of sketch and scroll methods for all users. We can see quite clearly that users strongly prefer the sketch method, with only two users rating the scroll method preferred, four showing no preference and the remainder (24) preferring the sketch method.

Qualitative examples are shown in figures 9a and 9b, showing the types of sketch created by the participants. We discuss further the difference between the types of sketch in Section 6 .

Finally, we reflect on the development of the precision of results across the session for users in the case more than one query was performed. Our purpose is to quantify the improvement between the first and the last query, without considering the cases in which the user found the target chair after the first interaction, and therefore considering the refinement of the results over time. We evaluate using the same mechanism as in the comparison of descriptors (sec. 5.1) but solely for the selected descriptor VGG-M, in fig. 7b (b) we can observe for each rank an improvement of the scores achieved by the last query compared with the first. To quantify this improvement we calculated the MAP for the first queries that achieves 0.17 , while the MAP for the last queries is 0.24 , showing an improvement during time.

\section{DISCUSSION}

In this section we discuss the outcomes achieved by the preliminary study on different descriptors and the results obtained by the user study.

\subsection{Comparison between 3D Sketch Descriptors}

Our preliminary study compares the precision achieved by different descriptors in order to decide the most accurate method for the user test. We defined the metric rules in such a way that it avoids assigning additional points if the target chair is present in the results. Despite of this, VGG-M clearly achieves highest precision scores for all the top 10 ranks. Consequently, this result shows that VGG-M descriptor is the most accurate in retrieving different facets (color, style and shape).

\subsection{User study}

Our purpose is to explore 3D sketch interaction for object retrieval in order to understand its validity and possible developments. Therefore, we designed an experiment to identify different user approaches between our method and a simple linear search. In addition, we avoided to include complex functionalities during sketch phase to study the effectiveness of pure sketch interaction.

Our experiment shows that it is possible, through an iterative process of sketching and model selection, to perform an effective search for a model in a large database while immersed in a virtual environment. Further the accuracy and the completion time are significantly better than a naive scroll method and the participants strongly preferred the sketch method over this naive method.

While the scroll method represents a baseline with a clear and linear work-flow to the user, the sketch method allows different strategies. In general two different techniques emerged from the experiment: sketch only as shown by examples in fig. $9 \mathrm{~b}$ and sketch with a model as shown in fig. 9a. The first and more intuitive approach is to make a single sketch and detail it step by step until most features of the chairs are resolved without replacing the model. The user can interrogate the system to have a feedback but essentially will continue to sketch. The downside is that the user can waste time on detailing a sketch and, in addition, can depict features that are not so relevant. Determining whether features are relevant or not is not a trivial task for two reasons. The first one is that different users will over-rate the saliency of the feature (e. g. it may be an uncommon feature but it hasn't been captured by the feature vector). The second one is the possibility that the specific feature is common to many objects of the database. In both cases the participant can experience an unsatisfactory answer from the system because it proposes a chair set without that feature or conversely many chairs containing it.

The second approach is to only model differences to the current object: that is the user queries the system and then only adds features that are different in the target object. The sketch is usually started again after each query. The advantage of this method is that the quick response from the system ( 2 seconds) enables fast iterative refinement. Every time the system receives a different combination of sketch and model it will retrieve a different set of chairs. This method requires more experience from the user, but 


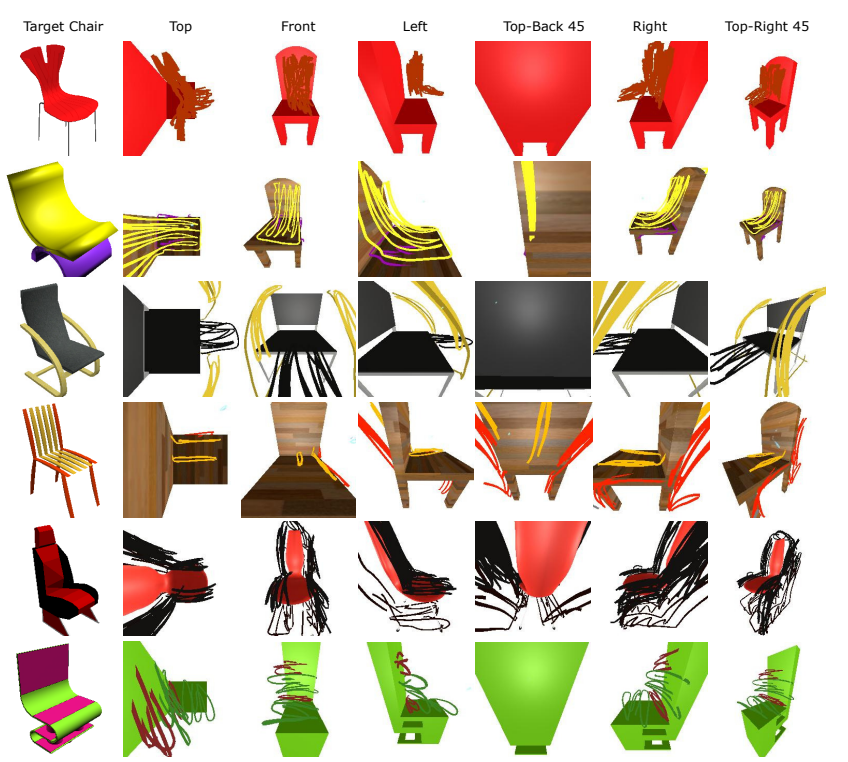

(a)
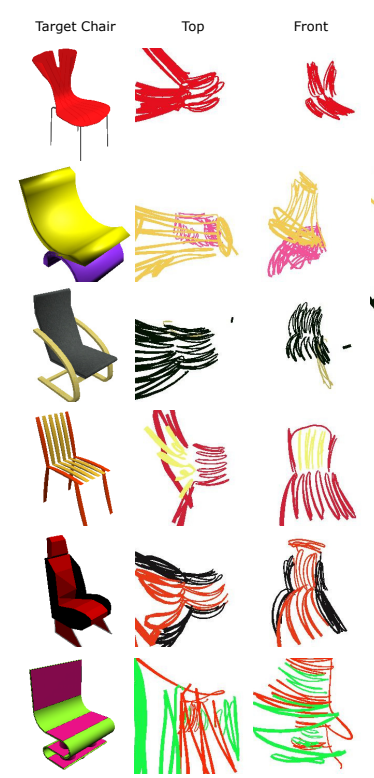
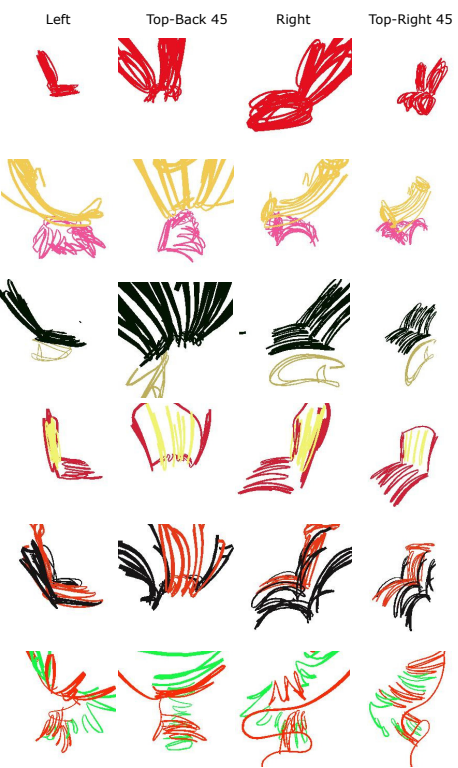

(b)

Figure 9: (a) Examples of users that successfully triggered the system using a combination of sketches and model. The left column contains the target chairs, while the other columns contain a subset of the snapshots used by the system. (b) Examples of users that successfully triggered the system using only sketches. The left column contains the target chairs, while the other columns contain a subset of the snapshots used by the system.

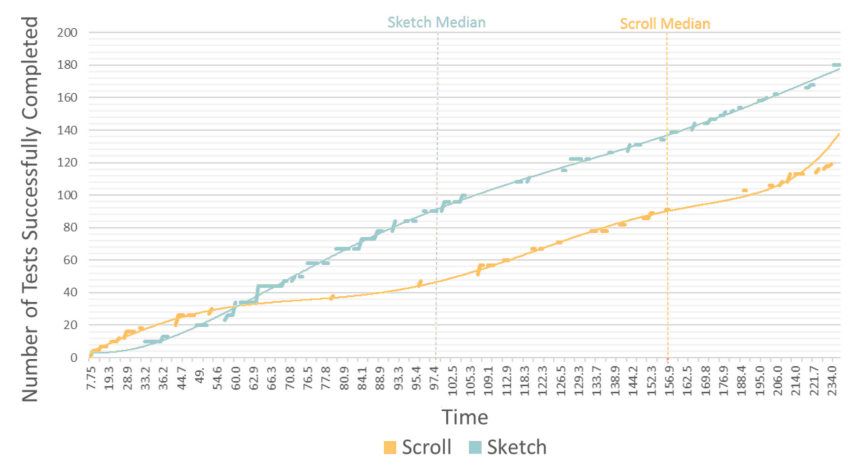

Figure 10: Cumulative time distribution for the scroll and sketch method. If the target chair was not found within the time limit (240 seconds) the time is limited to this.

after few iterations we observed several participants starting to adopt it.

In addition we demonstrate, through the comparison between first and last query outcomes, that user improves the precision as the search progresses with time, increasing the similarity of the facet of the retrieved models with the facet of the target.

\section{LIMITATIONS AND FUTURE WORK}

Despite the effectiveness of the use of sketch in this testing scenario and the positive responses during the user study, several aspects could be investigated to improve the search accuracy or interaction experience and in turn reduce interaction time. These are outlined below:

Multiple Object Categories In addition to working with chairs we performed an additional experiment with the table collection within the ShapeNet database. We verified the same behavior of the system using the same Multi-View descriptor and immersive retrieval approach. As the proposed approach has no fine-tune trained for the chair object category it is plausible results can further be extrapolated over the larger collection, with an initial object category selection at initialization.

Additional Descriptors In our preliminary study we compare different descriptors and only VGG-M belongs to the neural network algorithms. Recently, a considerable amount of neural network architectures is developed. We designed our system to facilitate the replacement of the descriptor generator. Therefore, a possible investigation is to extend the survey to the most promising CNNs. Gestures, Brushes and UI We opt to avoid additional interaction learning that can occur from gestures or brushes. But, these can be useful tools and allow a user to shortcut through tasks. It is easy to imagine using gesture recognition for object type identification (table etc.) avoiding the requirement for NLP or text selection. Or alternatively using familiar tools to photo editing software e.g. brushes to aid in depicting large region color, fill-bucket tool to specify a regions texture (an element not easily depicted).

Baseline method selection In this experiment we explored using linear search as the baseline, as it is a simple comparison that avoids taking the user out of the immersive reality or requiring additional 


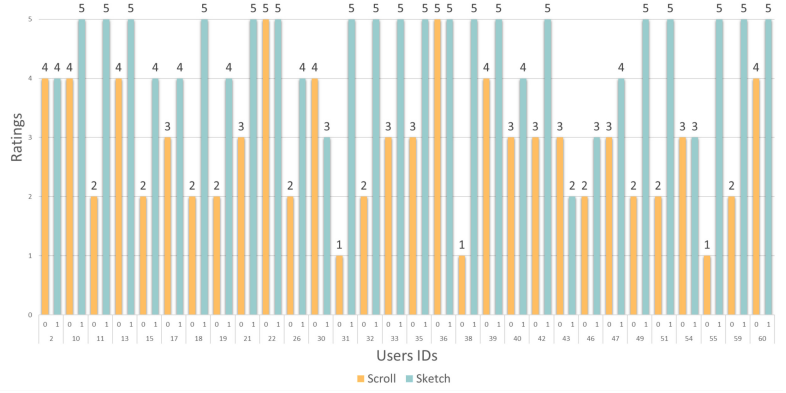

Figure 11: Each participants ratings for both the scroll and sketch method on a scale of one to five.

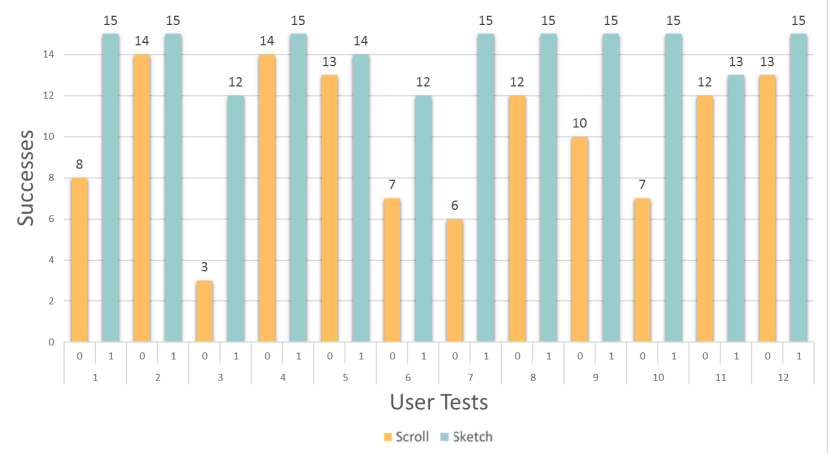

Figure 13: The number of successful searches made for scroll and sketch for each user.

training that could introduce bias. An alternative method of searching collections is based on text filtering or faceted search. Although an attractive approach, unfortunately model collections are frequently not annotated with rich adjectives to convey the visual appearance of the object. This is indeed the case with ShapeNet, even with the considerable amount of human annotation effort in terms of both keyword tags and brief descriptions these fail to describe the diversity of the model. This can be seen through fig. 12 where we analyze both tags and words in the description and show the top 150 keywords in a tag cloud scaled dependent on their frequency. Keywords often describe their specific object names, room or location that would be difficult or unlikely to be conveyed by the user (Meta-data for target models is provided in the supplementary material). It would be expected that a keyword search could only marginally improve search time, in the baseline case without a complex NLP.

\section{CONCLUSION}

The benefits of the virtual reality in the field of scene modeling have been investigated for several years. Previous research has focused on free-form modeling rather than developing a way to retrieve models from a large database. Current strategies for navigating an existing dataset use queries on tags or simply show to the user the

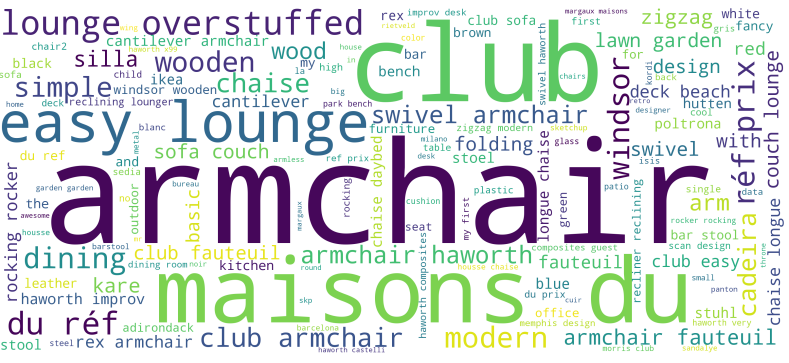

Figure 12: Word Cloud of the first 150 words frequency generated from tags and descriptions (with stopwords removed and chair removed). This is generated over the ShapeNet Chair subset.

entire set of models. In addition, large collections can suffer from a lack of meta-information which hampers model search and thus excludes part of the dataset from query results. We proposed a novel interaction paradigm that helps users to select a target item using an iterative sketch-based mechanism. We improve this interaction with the possibility of combining sketches and a background model together to form a query to search for a target model. We run a study to determine the most accurate descriptor. An experiment collected information about the time taken to complete the task and user experience rating. We compared our method with a naive scrolling selection method. The sketch-based method was clearly preferred by users and led to a significant reduction in search time. We thus believe that sketch-based queries are a very promising complement to existing immersive sketching systems.

\section{REFERENCES}

[1] Alaa E. Abdel-Hakim and Aly A. Farag. 2006. CSIFT: A SIFT Descriptor with Color Invariant Characteristics. In 2006 IEEE Computer Society Conference on Computer Vision and Pattern Recognition (CVPR 2006), 17-22 fune 2006, New York, NY, USA. 1978-1983. https://doi.org/10.1109/CVPR.2006.95

[2] Vandeborre J.P. Ansary T.F., Daoudi M. 2007. A Bayesian 3-D Search Engine Using Adaptive Views Clustering. IEEE Transaction on Multimedia 9, 1 (2007), 78 âĂŞ- 88 .

[3] Jonathan Ashley, Myron Flickner, James L. Hafner, Denis Lee, Wayne Niblack, and Dragutin Petkovic. 1995. The Query By Image Content (QBIC) System. In Proceedings of the 1995 ACM SIGMOD International Conference on Management of Data, San fose, California, May 22-25, 1995. 475. https://doi.org/10.1145/223784. 223888

[4] Martins N. V. Beatriz S. 2015. Air-Sketching for Object Retrieval. Technical Report. Instituto Superior T İAzecnico, Lisboa, Portugao.

[5] Alberto Del Bimbo, Pietro Pala, and Simone Santini. 1994. Visual Image Retrieval by Elastic Deformation of Object Sketches. In Proceedings IEEE Symposium on Visual Languages, St. Louis, Missouri, USA, October 4-7, 1994. 216-223. https: //doi.org/10.1109/VL.1994.363615

[6] Shinde J.V. Birari D.R. 2015. Survey on Sketch Based Image Retrieval. International fournal of Advanced Research in Computer and Communication Engineering 4, 12 (2015).

[7] Arthur W. Brody and Chris Hartman. 1999. BLUI: a body language user interface for 3D gestural drawing. In Human Vision and Electronic Imaging (SPIE Proceedings), Vol. 3644. SPIE, 356-363.

[8] Kimmel R. Bronstein M. A., Bronstein M. M. 2009. Topology-Invariant Similarity of Nonrigid Shapes. Int. F. Comput. Vis. 81 (2009), 281 âĂŞ- 301. https://doi.org/ 10.1007/s11263-008-0172-2

[9] T. Bui, L. Ribeiro, M. Ponti, and J. Collomosse. 2017. Compact descriptors for sketch-based image retrieval using a triplet loss convolutional neural network. Computer Vision and Image Understanding (2017). https://doi.org/10.1016/j.cviu. 2017.06.007

[10] Tu Bui, Leonardo Ribeiro, Moacir Ponti, and John P. Collomosse. 2016. Generalisation and Sharing in Triplet Convnets for Sketch based Visual Search. CoRR abs/1611.05301 (2016). 
[11] Jeff Butterworth, Andrew Davidson, Stephen Hench, and Marc. T. Olano. 1992 3DM: A Three Dimensional Modeler Using a Head-mounted Display. In Proceedings of the 1992 Symposium on Interactive 3D Graphics (I3D '92). ACM, New York, NY, USA, 135-138. https://doi.org/10.1145/147156.147182

[12] Abdolah Chalechale, Golshah Naghdy, and Alfred Mertins. 2005. Sketch-based image matching Using Angular partitioning. IEEE Trans. Systems, Man, and Cybernetics, Part A 35, 1 (2005), 28-41. https://doi.org/10.1109/TSMCA.2004. 838464

[13] K. Chatfield, K. Simonyan, A. Vedaldi, and A. Zisserman. 2014. Return of the Devil in the Details: Delving Deep into Convolutional Nets. In British Machine Vision Conference. arXiv:cs/1405.3531

[14] Han-wool Choi, Hee-joon Kim, Jeong-in Lee, and Young-Ho Chai. 2005. Free Hand Stroke Based Virtual Sketching, Deformation and Sculpting of NURBS Surface. In Proceedings of the 2005 International Conference on Augmented Tele-existence (ICAT '05). ACM, New York, NY, USA, 3-9. https://doi.org/10.1145/1152399.1152401

[15] James H. Clark. 1976. Designing Surfaces in 3-D. Commun. ACM 19, 8 (Aug 1976), 454-460. https://doi.org/10.1145/360303.360329

[16] John P. Collomosse, Graham McNeill, and Leon Adam Watts. 2008. Free-hand sketch grouping for video retrieval. In ICPR. IEEE Computer Society, 1-4.

[17] Navneet Dalal and Bill Triggs. 2005. Histograms of Oriented Gradients for Human Detection. In 2005 IEEE Computer Society Conference on Computer Vision and Pattern Recognition (CVPR 2005), 20-26 June 2005, San Diego, CA, USA. 886-893. https://doi.org/10.1109/CVPR.2005.177

[18] Michael F. Deering. 1996. The HoloSketch VR Sketching System. Commun. ACM 39, 5 (May 1996), 54-61. https://doi.org/10.1145/229459.229466

[19] Mathias Eitz, Kristian Hildebrand, Tamy Boubekeur, and Marc Alexa. 2011 Sketch-Based Image Retrieval: Benchmark and Bag-of-Features Descriptors. IEEE Transactions on Visualization and Computer Graphics 17, 11 (2011), 1624-1636.

[20] Mathias Eitz, Ronald Richter, Tamy Boubekeur, Kristian Hildebrand, and Marc Alexa. 2012. Sketch-based shape retrieval. ACM Trans. Graph. 31, 4 (2012), 31:1-31:10. https://doi.org/10.1145/2185520.2185527

[21] Alexa M. Eitz M., Hays J. 2012. How Do Humans Sketch Objects?. In ACM Trans. Graphics, 31(4).

[22] Michele Fiorentino, Giuseppe Monno, Pietro Renzulli, and Antonio Uva. 2003. 3D sketch stroke segmentation and fitting in virtual reality. (01 2003).

[23] M. J. Fonseca, S. James, and J. Collomosse. 2012. Skeletons from sketches of dancing poses. In 2012 IEEE Symposium on Visual Languages and Human-Centric Computing (VL/HCC). 247-248. https://doi.org/10.1109/VLHCC.2012.6344537

[24] Thomas Funkhouser, Patrick Min, Michael Kazhdan, Joyce Chen, Alex Halderman, David Dobkin, and David Jacobs. 2003. A Search Engine for 3D Models. ACM Trans. Graph. 22, 1 (Jan. 2003), 83-105. https://doi.org/10.1145/588272.588279

[25] Rui Hu and John Collomosse. 2013. A Performance Evaluation of Gradient Field HOG Descriptor for Sketch Based Image Retrieval. Comput. Vis. Image Underst. 117, 7 (July 2013), 790-806. https://doi.org/10.1016/j.cviu.2013.02.005

[26] Stuart James and John Collomosse. 2014. Interactive Video Asset Retrieval Using Sketched Queries. In Proceedings of the 11th European Conference on Visual Media Production (CVMP '14). ACM, New York, NY, USA, Article 11, 8 pages. https://doi.org/10.1145/2668904.2668940

[27] Ricardo Jota, Alfredo Ferreira, Mariana Cerejo, José Santos, Manuel J. Fonseca and Joaquim A. Jorge. 2006. Recognizing Hand Gestures with CALI. In SIACG. Eurographics Association, 187-193.

[28] Daniel F. Keefe, Daniel Acevedo Feliz, Tomer Moscovich, David H. Laidlaw, and Joseph J. LaViola, Jr. 2001. CavePainting: A Fully Immersive 3D Artistic Medium and Interactive Experience. In Proceedings of the 2001 Symposium on Interactive 3D Graphics (I3D '01). ACM, New York, NY, USA, 85-93. https: //doi.org/10.1145/364338.364370

[29] Daniel F. Keefe, Robert C. Zeleznik, and David H. Laidlaw. 2007. Drawing on Air Input Techniques for Controlled 3D Line Illustration. 13, 5 (2007), 1067-1081.

[30] Yann Lecun, LÃlon Bottou, Yoshua Bengio, and Patrick Haffner. 1998. Gradientbased learning applied to document recognition. In Proceedings of the IEEE. 22782324.

[31] Yong Jae Lee, C. Lawrence Zitnick, and Michael F. Cohen. 2011. ShadowDraw: real-time user guidance for freehand drawing. ACM Trans. Graph. 30, 4 (2011), 27:1-27:10.

[32] Biao Leng, Yu Liu, Kai Yu, Xiangyang Zhang, and Zhang Xiong. 2016. 3D Object Understanding with 3D Convolutional Neural Networks. Inf. Sci. 366, C (Oct. 2016), 188-201. https://doi.org/10.1016/j.ins.2015.08.007

[33] Bo Li, Yijuan Lu, Fuqing Duan, Shuilong Dong, Yachun Fan, Lu Qian, Hamid Laga, Haisheng Li, Yuxiang Li, Peng Liu, Maks Ovsjanikov, Hedi Tabia, Yuxiang Ye, Huanpu Yin, and Ziyu Xue. 2016. 3D Sketch-Based 3D Shape Retrieval. In Eurographics Workshop on 3D Object Retrieval, A. Ferreira, A. Giachetti, and D. Giorgi (Eds.). The Eurographics Association. https://doi.org/10.2312/3dor 20161087

[34] David G. Lowe. 2004. Distinctive Image Features from Scale-Invariant Keypoints. International fournal of Computer Vision 60, 2 (01 Nov 2004), 91-110. https //doi.org/10.1023/B:VISI.0000029664.99615.94
[35] Gen Nishida, Ignacio Garcia-Dorado, Daniel G. Aliaga, Bedrich Benes, and Adrien Bousseau. 2016. Interactive Sketching of Urban Procedural Models. ACM Trans. Graph. 35, 4, Article 130 (July 2016), 11 pages. https://doi.org/10.1145/2897824. 2925951

[36] Robert Osada, Thomas Funkhouser, Bernard Chazelle, and David Dobkin. 2002. Shape Distributions. ACM Transactions on Graphics 21 (2002), 807-832.

[37] Yonggang Qi, Yi-Zhe Song, Honggang Zhang, and Jun Liu. 2016. Sketch-based image retrieval via Siamese convolutional neural network. In 2016 IEEE International Conference on Image Processing, ICIP 2016, Phoenix, AZ, USA, September 25-28, 2016. 2460-2464. https://doi.org/10.1109/ICIP.2016.7532801

[38] Dominik Rausch, Ingo Assenmacher, and Torsten W. Kuhlen. 2010. 3D Sketch Recognition for Interaction in Virtual Environments. In Proceedings of the Seventh Workshop on Virtual Reality Interactions and Physical Simulations, VRIPHYS 2010, Copenhagen, Denmark, 2010. 115-124. https://doi.org/10.2312/PE/vriphys/ vriphys10/115-124

[39] David Gavilan Ruiz, Suguru Saito, and Masayuki Nakajima. 2007. Sketch-tocollage. In SIGGRAPH Posters. ACM, 35

[40] Raif M. Rustamov. 2010. Robust Volumetric Shape Descriptor. In 3DOR. Eurographics Association, 1-5.

[41] Patsorn Sangkloy, Nathan Burnell, Cusuh Ham, and James Hays. 2016. The sketchy database: learning to retrieve badly drawn bunnies. ACM Trans. Graph. 35, 4 (2016), 119:1-119:12.

[42] Tiago Santos, Alfredo Ferreira, Filipe Dias, and Manuel J. Fonseca. 2008. Using Sketches and Retrieval to Create LEGO Models. In Proceedings of the Fifth Eurographics Conference on Sketch-Based Interfaces and Modeling (SBM'08). Eurographics Association, Aire-la-Ville, Switzerland, Switzerland, 89-96. https: //doi.org/10.2312/SBM/SBM08/089-096

[43] Steven Schkolne, Michael Pruett, and Peter Schröder. 2001. Surface Drawing: Creating Organic 3D Shapes with the Hand and Tangible Tools. In Proceedings of the SIGCHI Conference on Human Factors in Computing Systems (CHI '01). ACM, New York, NY, USA, 261-268. https://doi.org/10.1145/365024.365114

[44] Eugenio Di Sciascio, G. Mingolla, and Marina Mongiello. 1999. Content-Based Image Retrieval over the Web Using Query by Sketch and Relevance Feedback. In VISUAL (Lecture Notes in Computer Science), Vol. 1614. Springer, 123-130.

[45] HyoJong Shin and Takeo Igarashi. 2007. Magic Canvas: Interactive Design of a 3-D Scene Prototype from Freehand Sketches. In Proceedings of Graphics Interface 2007 (GI '07). ACM, New York, NY, USA, 63-70. https://doi.org/10.1145/1268517. 1268530

[46] Pedro Manuel Antunes Sousa and Manuel J. Fonseca. 2010. Sketch-based retrieval of drawings using spatial proximity. 7. Vis. Lang. Comput. 21, 2 (2010), 69-80. https://doi.org/10.1016/j.jvlc.2009.12.001

[47] Hang Su, Subhransu Maji, Evangelos Kalogerakis, and Erik Learned-Miller. 2015. Multi-view Convolutional Neural Networks for 3D Shape Recognition. In Proceedings of the 2015 IEEE International Conference on Computer Vision (ICCV) (ICCV '15). IEEE Computer Society, Washington, DC, USA, 945-953. https://doi.org/10.1109/ICCV.2015.114

[48] Hang Su, Subhransu Maji, Evangelos Kalogerakis, and Erik G. Learned-Miller. 2015. Multi-view convolutional neural networks for $3 \mathrm{~d}$ shape recognition. In Proc. ICCV.

[49] Fang Wang, Le Kang, and Yi Li. 2015. Sketch-Based 3D Shape Retrieval Using Convolutional Neural Networks. In The IEEE Conference on Computer Vision and Pattern Recognition (CVPR).

[50] Jiang Wang, Yang Song, Thomas Leung, Chuck Rosenberg, Jingbin Wang, James Philbin, Bo Chen, and Ying Wu. 2014. Learning Fine-grained Image Similarity with Deep Ranking. CoRR abs/1404.4661 (2014).

[51] Gerold Wesche and Hans-Peter Seidel. 2001. FreeDrawer: A Free-form Sketching System on the Responsive Workbench. In Proceedings of the ACM Symposium on Virtual Reality Software and Technology (VRST '01). ACM, New York, NY, USA, 167-174. https://doi.org/10.1145/505008.505041

[52] Zhirong Wu, Shuran Song, Aditya Khosla, Fisher Yu, Linguang Zhang, Xiaoou Tang, and Jianxiong Xiao. 2015. 3D ShapeNets: A deep representation for volumetric shapes. In CVPR. IEEE Computer Society, 1912-1920.

[53] Kun Xu, Kang Chen, Hongbo Fu, Wei-Lun Sun, and Shi-Min Hu. 2013. Sketch2Scene: Sketch-based Co-retrieval and Co-placement of 3D Models. ACM Transactions on Graphics 32, 4 (2013), 123:1-123:12.

[54] Yongxin Yang and Timothy M. Hospedales. 2015. Deep Neural Networks for Sketch Recognition. CoRR abs/1501.07873 (2015). http://arxiv.org/abs/1501.07873

[55] Q. Yu, F. Liu, Y. Z. Song, T. Xiang, T. M. Hospedales, and C. C. Loy. 2016. Sketch Me That Shoe. In 2016 IEEE Conference on Computer Vision and Pattern Recognition (CVPR). 799-807. https://doi.org/10.1109/CVPR.2016.93 